\title{
Avaliação da qualidade do solo em agroecossistemas na zona rural de São Bentinho- PB
}

\section{Evaluation of soil quality in agroecosystems in rural São Bentinho-PB}

\author{
Maria de Fátima Araújo Alves ${ }^{1 *}$, Silvana Nóbrega Ribeiro ${ }^{2}$, Francisca Jéssica da Silva Melo ${ }^{3}$, Lígia Rejane Araújo Alves ${ }^{4}$ \\ Zacarias Caetano Vieira ${ }^{5}$
}

Resumo: O crescimento da demanda por alimentos gera a expansão de áreas agrícolas ocasionando um aumento do desmatamento. Tais fatores contribuem para o crescimento da preocupação com a sustentabilidade dos sistemas agrícolas, o qual passa por uma percepção do espaço rural como agente ativo na conservação e proteção dos recursos naturais. $\mathrm{O}$ conhecimento dos limites de exploração dos ecossistemas rurais é fator primordial para que se obtenham sistemas agrícolas mais sustentáveis. Diante do exposto o presente trabalho objetiva avaliar, através de uma extensa revisão bibliográfica, visitas em campo e utilização de índices de sustentabilidade, a qualidade do solo de dois agroecossistemas utilizados para o plantio de feijão e milho na zona rural de São Bentinho-PB; e finalmente, propor estratégias ou técnicas de manejo que venham tornar os agroecossistemas estudados mais sustentáveis. Conclui-se que a área de plantio de feijão apresentou um solo de melhor qualidade do que área de plantio de milho. A utilização dessa metodologia para avaliar a qualidade do solo mostrou-se simples e acessível, podendo direcionar as ações para uma agricultura mais sustentável, necessitando-se para isso, qualificação e treinamento dos agricultores. Finalmente, deve-se treinar esses pequenos produtores também na aplicação de técnicas sustentáveis de manejo do solo.

Palavras-chaves: sustentabilidade, agricultura, percepção

\begin{abstract}
The growth in demand for food generates the expansion of agricultural areas causing an increase in deforestation. Such factors contribute to the growing concern about the sustainability of agricultural systems, which involves a perception of the countryside as an active agent in the conservation and protection of natural resources. Knowledge of rural ecosystems operating limits is a key factor in order to obtain more sustainable agricultural systems. Given the above the present study aims to evaluate, through an extensive literature review, field visits and use of sustainability indexes, the quality of the two agro soil used for planting beans and corn in the countryside of São Bento-PB; and finally, propose strategies and management techniques that may make them more sustainable agro-ecosystems studied. In conclusion, the bean planting area presented a solo better quality than corn planting area. The use of this methodology for assessing soil quality proved to be simple and affordable, and can direct the actions for a more sustainable agriculture, requiring up to that, qualifications and training of farmers. Finally, one should train these small producers also in the implementation of sustainable techniques for soil management.
\end{abstract}

Key words: sustainability, agriculture, perception.

\footnotetext{
*Autor para correspondência

Recebido para publicação em 23/08/2015; aprovado em 10/12/2015

${ }_{1}^{1}$ Graduada em Engenharia Ambiental, UFCG, Pombal-PB; (83) 9633-3929, mariaufcg2013@ gmail.com.

${ }^{2}$ Graduanda em Engenharia Ambiental, UFCG, silvnanobreri@ hotmail.com

${ }^{3}$ Graduanda em Engenharia Ambiental, UFCG, jessicahmello90@gmail.com

${ }^{4}$ Graduanda em Engenharia Ambiental, UFCG, ligia.rejane@ yahoo.com.br

${ }^{5}$ Graduado em Engenharia Civil. Mestre em Engenharia Civil e Ambiental (UFCG). Professor do Instituto Federal de Sergipe, (79) 9146-1619,

zacariascaetano@gmail.com.
} 


\section{INTRODUÇÃO}

Diante da necessidade de uma interação harmônica entre homem e natureza surgiu às discussões em torno da temática da sustentabilidade (OLIVEIRA, 2007). A sustentabilidade passou a ser uma necessidade no desenvolvimento nos últimos anos, inserindo-se dentro da produção agrícola com o objetivo de reduzir a exploração dos recursos naturais mantendo o nível de produção (CARVALHO, 2007).

$\mathrm{O}$ uso da ciência e de novas técnicas na agricultura substitui o conhecimento empírico atribuindo um novo modelo de manejo dos recursos naturais, fazendo com que a natureza fosse dominada pelo homem de uma forma mais sustentável (GUZMÀN, 2009). Entretanto, vale salientar que o conhecimento acumulado ao longo dos anos por agricultores não deve ser desconsiderado, pois segundo Altieri (2012) o conjunto de práticas agrícolas empregadas por muitos agricultores tradicionais representa, sem dúvida, uma rica fonte de saberes para os trabalhadores atuais que procuram criar agroecossistemas inovadores que se adaptem às condições agroecológicas e socioeconômicas locais.

No pensamento de Gliessman (2003), para que se tenham sistemas agrícolas mais sustentáveis é necessário compreender que cada ecossistema tem um limite para produção, e deve-se levar em consideração a capacidade produtiva do solo, a qualidade e a quantidade dos alimentos que precisa para atender a demanda. Dentro desse contexto, os índices e os indicadores de sustentabilidade têm sido utilizados como importantes ferramentas no processo de planejamento e gestão de uma agricultura cada vez mais sustentável.

Atualmente, diversos índices/indicadores de qualidade do solo são obtidos com o auxílio de equipamentos específicos e por meio de análises laboratoriais, porém, metodologias como a de Altieri e Nicholls (2002), embora sendo subjetiva, constitui-se numa importante alternativa para avaliação da qualidade do solo, sobretudo em regiões em que predominam pequenos agricultores, já que geralmente estes dispõem de poucos recursos além do fato de equipamentos e laboratórios serem de difícil acesso.

Nesse sentido, esta pesquisa tem por objetivo avaliar por meio dos índices de sustentabilidade citados na metodologia de Altieri e Nicholls (2002), a qualidade do solo de dois agroecossistemas utilizados para o plantio de feijão e milho na zona rural de São Bentinho-PB, e posteriormente, propor estratégias ou técnicas de manejo que venham tornar os agroecossistemas estudados mais sustentáveis

\section{MATERIAL E MÉTODOS}

\section{Área de Estudo}

Para realização dessa pesquisa foram escolhidas duas áreas de plantios (Tabela 1), com culturas distintas. Em ambas as áreas são utilizadas os monocultivos, e após a colheita, os animais são soltos na roça para se alimentarem dos restos das plantações. Essas áreas estão inseridas no munícipio de São Bentinho - PB localizado no Oeste do Estado da Paraíba (Figura 1). Segundo Beltrão et al. (2005) o município de São Bentinho, está inserido na unidade geoambiental da Depressão Sertaneja, que representa a paisagem típica do semiárido nordestino, tendo sua vegetação basicamente composta por Caatinga Hiperxerófila com trechos de Floresta Caducifólia, e clima do tipo Tropical Semiárido, com chuvas de verão. O período chuvoso se inicia em fevereiro com término em abril. A precipitação média anual é de $431,8 \mathrm{~mm}$.

Tabela 1 - Dados das roças escolhidas para realização de estudo

\begin{tabular}{cccc}
\hline Roça & Plantio & Área (ha) & Pessoas \\
\hline 1 & Feijão & 2 & 5 \\
2 & Milho & 2 & 7
\end{tabular}

Figura 1 - Localização do Município de São Bentinho no Estado da Paraíba-

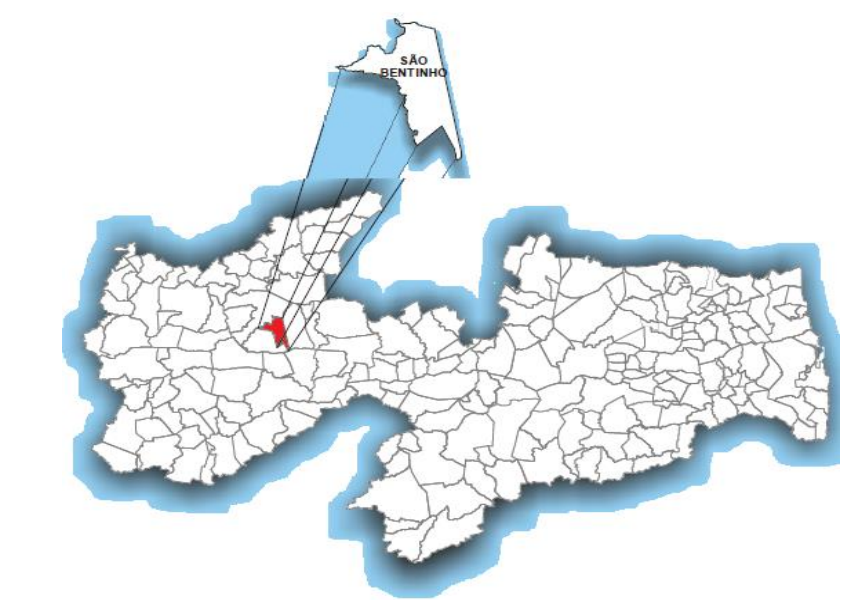

Fonte: Adaptado de Beltrão et al. (2005)

\section{Metodologia}

Foram realizadas visitas de campo para diagnosticar dentro das áreas de plantios e nas áreas de entorno a presença de resíduos agroindustriais e restos de plantações como palhas de milho, cascas de legumes e de frutas. Após o diagnóstico, foi realizado o levantamento de dados sobre as culturas inseridas no local em anos anteriores por meio de conversas informais com as famílias de agricultores, totalizando doze pessoas entrevistadas. Posteriormente, foi analisada a qualidade dos solos através de índices de sustentabilidade adaptados as condições locais. E finalmente, realizou-se a conscientização dos agricultores.

\section{Índices de sustentabilidade}

Para a análise da qualidade do solo foram utilizados os índices de sustentabilidade para agroecossistemas citados na metodologia de Altieri e Nicholls (2002) e adaptadas para as condições locais, os quais para Nicholls et al. (2004) executam o seu objetivo de forma simples e rápida. Essa metodologia trata de questões sobre a qualidade do solo, busca informações a respeito dos diversos indicadores estudados com base no conhecimento dos agricultores. Para sua aplicação organizou-se uma tabela, onde foram escolhidos 9 indicadores contendo as características desejadas para o estudo, as quais estão sintetizadas abaixo (Tabela 02). 
Tabela 02 - Características observadas para a avaliação da qualidade do solo em agroecossistemas na zona rural de São Bentinho-PB

\begin{tabular}{|c|c|c|c|}
\hline Itens & Indicador & Valor & Características observadas \\
\hline \multirow[t]{3}{*}{1} & Erosão & $1-4$ & Erosão severa; \\
\hline & & $5-9$ & Presença de sulcos; \\
\hline & & 10 & Sem sinais visíveis de erosão. \\
\hline \multirow[t]{3}{*}{2} & Cor & $1-4$ & Coloração clara/ amarela; \\
\hline & & $5-9$ & Vermelho/Amarelo; \\
\hline & & 10 & Escuro. \\
\hline \multirow[t]{3}{*}{3} & Cobertura do solo & $1-4$ & Solo sem nenhuma palha; \\
\hline & & $5-9$ & Fina camada de palha; \\
\hline & & 10 & Solo bem coberto com restos vegetais. \\
\hline \multirow[t]{3}{*}{4} & Crescimento e aparência das & $1-4$ & Vegetação baixa, fina e amarelada; \\
\hline & plantas & $5-9$ & Vegetação amarelada, médio porte; \\
\hline & & 10 & Vegetação verde escura, porte alto. \\
\hline \multirow[t]{3}{*}{5} & Compactação & $1-4$ & A cavadeira perfura com dificuldade; \\
\hline & & $5-9$ & A cavadeira entra com menos dificuldade; \\
\hline & & 10 & A cavadeira entra com facilidade; \\
\hline \multirow[t]{3}{*}{6} & Textura & $1-4$ & Arenoso; \\
\hline & & $5-9$ & Argiloso; \\
\hline & & 10 & Arenoso/Argiloso. \\
\hline \multirow[t]{3}{*}{7} & Vegetação & $1-4$ & Ausência de plantas; \\
\hline & & $5-9$ & Presença de plantas; \\
\hline & & 10 & Com muitas plantas. \\
\hline \multirow[t]{2}{*}{8} & Estrutura & $1-4$ & Desmancha fácil quando se coloca na mão; \\
\hline & & $\begin{array}{c}5-9 \\
10\end{array}$ & $\begin{array}{c}\text { Não se desmancha tão fácil precisa de uma pressão; } \\
\text { Difícil de ser quebrado, não desmancha. }\end{array}$ \\
\hline \multirow[t]{2}{*}{9} & Atividade biológica & $1-4$ & Sem minhocas ou outros invertebrados do solo; \\
\hline & & $\begin{array}{c}5-9 \\
10\end{array}$ & Presença de algumas minhocas ou outros invertebrados do solo; \\
\hline
\end{tabular}

Fonte: Adaptada de Gervazio et al. (2014).

\section{Características das áreas}

Foram coletados junto aos agricultores dados das áreas estudadas. Eles analisaram visualmente, as características, e em seguida, atribuíram uma nota que variava de 1(um) a 10 (dez), e quando uma mesma característica recebia notas diferentes, as dúvidas eram esclarecidas havendo uma concordância entre os agricultores. As médias foram calculadas e comparadas com os valores apresentados por Machado (2006), que cita uma faixa de valores para a indicação da sustentabilidade, onde valores médios inferiores a 5, significa que a área apresenta sustentabilidade mínima. Com as médias calculadas foram plotados gráficos do tipo radar no programa Microsoft Excel 2010 para melhor visualizar esses indicadores. Para Rigo e Silva (2011) nesse gráfico, os pontos ligados, revelam que quanto mais próximo for da margem do círculo, mais sustentável é o sistema. Posteriormente foi somado cada média e dividida pelo total dos números de indicadores para quantificar a nota geral de cada agroecossistema.

\section{RESULTADOS E DISCUSSÃO}

\section{Diagnóstico da área}

Em relação à presença de algum resíduo tóxico na área de plantio e no entorno não foram relatados nenhum vestígio agroindustrial, apenas material orgânico como resto de palhas e estercos de animais. A vegetação de entorno encontra-se conservada e segundo Machado (2006) serve de barreira física contra os ventos e contaminantes, de habitats para possíveis inimigos naturais. Próxima a área 1 , há $30 \mathrm{~m}^{2}$ plantados com batata, e por ser uma área mais encharcada, não foi considerada nesse trabalho.

\section{Dados dos indicadores da área 1}

Na tabela 3 e figura 3 encontram-se as notas atribuídas a cada um dos índices de sustentabilidade do solo na área 1. A maior nota foi para o parâmetro erosão, na qual não foi relatado nenhum vestígio e a menor nota foi para a compactação do solo, o que segundo Nicholls et al. (2004) indica que a área necessita de correções agroecológicas para corrigir a deficiência do solo, nas culturas ou até mesmo nos sistemas.

Figura 2 - Agroecossistema plantado com feijão

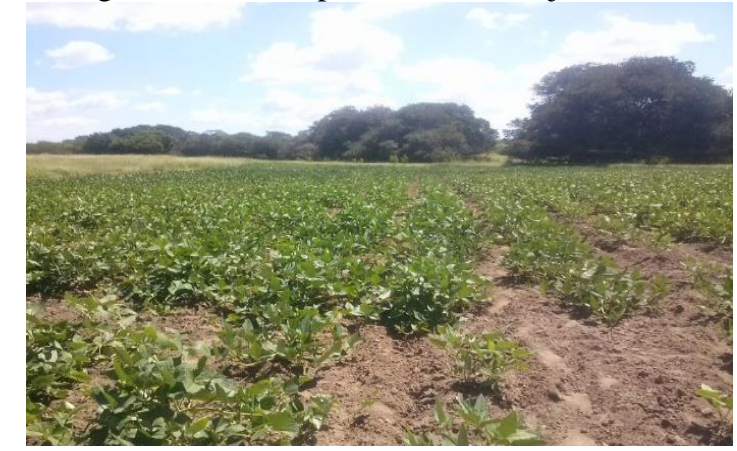

Fonte: Autor Principal 
Tabela 03 - Notas dos indicadores de acordo com a percepção dos agricultores na área de feijão

\begin{tabular}{llcl}
\hline Itens & Indicador & Notas & \multicolumn{1}{c}{ Características observadas } \\
\hline $\mathbf{1}$ & Erosão & 10 & Sem sinais visíveis de erosão \\
$\mathbf{2}$ & Cor & 8 & Vermelho/Amarelo \\
$\mathbf{3}$ & Cobertura do solo & 8 & Fina camada de palha \\
$\mathbf{4}$ & Crescimento e aparência das plantas & 8 & Vegetação de médio porte \\
$\mathbf{5}$ & Compactação & 5 & A cavadeira perfura com menos dificuldade \\
$\mathbf{6}$ & Textura & 8 & Argiloso \\
$\mathbf{7}$ & Vegetação & 8 & Presença de plantas \\
$\mathbf{8}$ & Estrutura & 7 & Não se desmancha tão fácil, precisa de uma pressão \\
$\mathbf{9}$ & Atividade biológica & 8 & Presença minhocas ou invertebrados do solo \\
\hline
\end{tabular}

O valor médio para a qualidade do solo dessa propriedade foi de 7,7. A compactação foi à característica que mais deixou a desejar. Tal fato atribui-se a presença de animais na área após o plantio, que conforme Kochhann (2000) pode resultar de forças mecânicas, pisoteio de animais e/ou percolação da água sobre o solo. Esse processo de compactação pode prejudicar a área vizinha plantada com batata. Esse fato deve-se à taxa de infiltração da água ser restringida em solos compactados, resultando-se em uma acumulação (encharcamento) no local das raízes das plantas depois das chuvas ou em irrigações (COPAS et al., 2009). Esse acúmulo de água segundo Thornton et al. (2008) na cultura da batata, causa a redução do fruto e afeta a qualidade das batatas, além de acelerar o dano ocasionado por doenças.

Figura 3 - Reprodução esquemática dos indicadores de qualidade do solo para a área 1

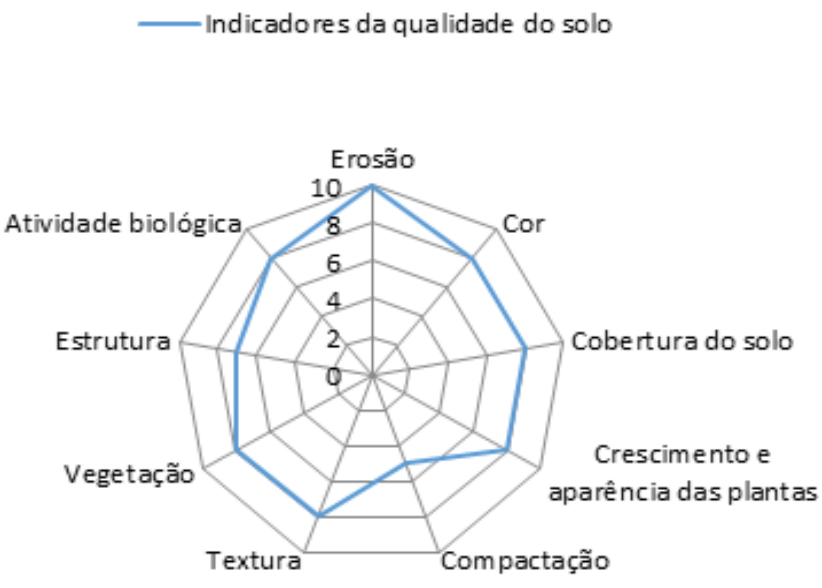

\section{Dados dos indicadores da área 2}

Na tabela 4 e figura 4 encontram-se as notas atribuídas a cada um dos índices de sustentabilidade do solo na área 2 utilizados para avaliação de sua qualidade. Observa-se que a maior nota, assim como na área 1 , foi obtida pelo indicador erosão. A menor nota obtida foi pelo indicador cor, justificada pela apresentação de uma coloração avermelhada, indicando que a mesma apresenta um solo com pouca matéria orgânica. A nota média do agroecossistema 2 foi de 7,5.

Analisando os dois agroecossistemas de forma geral, percebe-se que a área 1 é mais sustentável que a 2, e que ambas as áreas podem ser melhoradas para uma maior conservação do solo e melhores resultados da produção.

Figura 4 - Reprodução esquemática dos indicadores de qualidade do solo para a área 2

- Indicadores da qualidade do solo

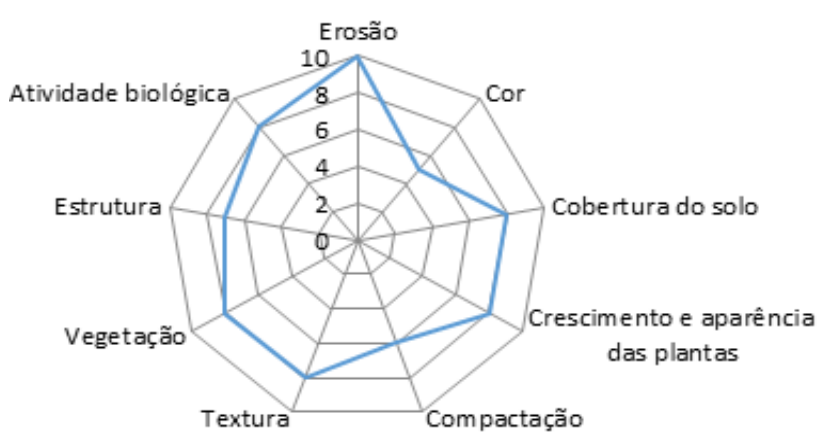

Figura 5 - Agroecossistema plantado com milho

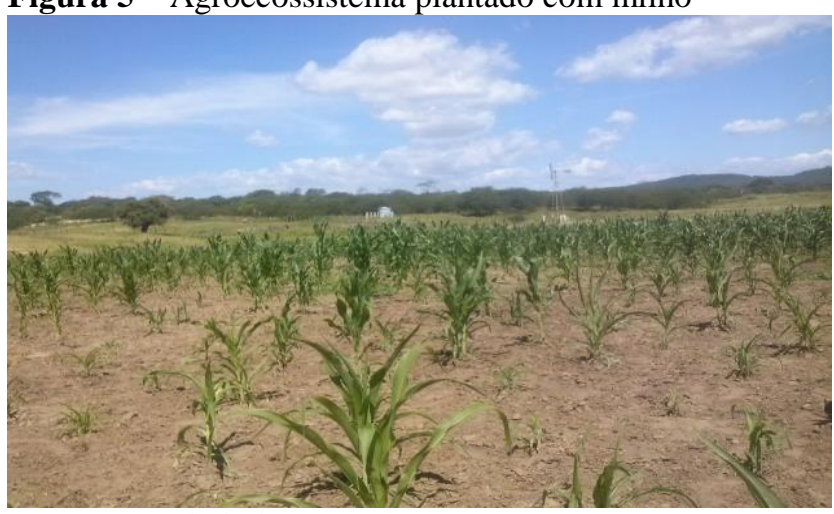

Fonte: Autor Principal 
Tabela 04 - Notas dos indicadores de acordo com a percepção dos agricultores na área de milho

\begin{tabular}{rlrl}
\hline Itens & Indicador & Notas & Características observadas \\
\hline $\mathbf{1}$ & Erosão & 1 & Vestígio de erosão \\
$\mathbf{2}$ & Cor & 5 & Esbranquiçada \\
$\mathbf{3}$ & Cobertura do solo & 8 & Algum resto de palha \\
$\mathbf{4}$ & Crescimento e aparência das plantas & 8 & Vegetação de baixo porte \\
$\mathbf{5}$ & Compactação & 6 & Menor dificuldade de perfuração \\
$\mathbf{6}$ & Textura & 8 & Argiloso \\
$\mathbf{7}$ & Vegetação & 8 & Presença de plantas \\
$\mathbf{8}$ & Estrutura & 7 & Solo com torrões duros, \\
$\mathbf{9}$ & Atividade biológica & 8 & Minhocas e invertebrados do solo \\
\hline
\end{tabular}

Altieri (1994) cita que para manter agroecossistemas mais sustentáveis, antes de tudo deve se observar a capacidade de suporte do solo e fazer uso da implantação de mais de um cultivo, assim, a área estaria mais forte em relação ao surgimento de pragas, os agricultores aproveitariam mais a propriedade e teriam mais alimentos e menos gastos com agrotóxicos. Nicholls et al. (1999) acredita que os sistemas monocultivos reduz a capacidade da área de conter mecanismos ecológicos capazes de inibir a presença de pragas. Dessa forma, a lavoura fica mais susceptível ao ataque de doenças e de insetos, podendo resultar em perdas econômicas para os lavradores.

Além da rotação de cultura Altieri e Nicholls (2002) citam que há muitas outras ações que podem ser desenvolvidas em prol da sustentabilidades dos agroecossistemas como o plantio direto, quebra-ventos, cercas vivas, barreiras de cata-vento e a adoção de programas educativos voltados para conscientização dos agricultores, visto que estes tem dificuldades de lidar com questões ambientais devido à falta de políticas públicas especificas que visem ensinar e conscientizar os trabalhadores rurais na temática da sustentabilidade, bem como incentiva-los na adoção de tais medidas.

\section{CONCLUSÕES}

Conclui-se que a área de plantio de feijão apresentou um solo de melhor qualidade do que a área de plantio de milho, visto que as técnicas utilizadas nesse roçado são mais sustentáveis. A utilização dessa metodologia para avaliar a qualidade do solo mostrou-se simples e acessível, podendo direcionar as ações para uma agricultura mais sustentável, servindo de parâmetro para tomadas de decisão, necessitandose para isso, treinar e qualificar esses pequenos produtores, não apenas no uso dessa metodologia, mas também na aplicação de técnicas sustentáveis de manejo do solo.

\section{REFERÊNCIAS BIBLIOGRÁFICAS}

ALTIERI, M. Agroecologia: bases científicas para uma agricultura sustentável. $3^{\mathrm{a}}$ ed. rev. ampl. São Paulo: Expressão Popular; AS-PTA, 2012.

ALTIERI, M. A. O papel ecológico da biodiversidade em agroecossistemas: alternativas. Cadernos de Agroecologia: biodiversidade, Rio de Janeiro, p. 1-6, 1994.
ALTIERI, M. A.; NICHOLLS, C.I. Un método agroecologico rapido para la evaluación de la sostenibilidad de cafetales. Manejo Integrado de Plagas y Agroecologia, Turrialba, v. 64, p.17-24, 2002.

BELTRÃO, B. A.; MORAIS, F.; MASCARENHAS, J. C.; MIRANDA, J. L. F.; JUNIOR, L. C. S.; MENDES, V. A. Projeto cadastro de fontes de abastecimento por água subterrânea. Diagnóstico do município de Pombal, estado da Paraíba. CPRM - Serviço Geológico do Brasil. Recife, 2005.

CARVALHO, H. M. Impactos econômicos, sociais e ambientais devidos á expansão da oferta do etanol no Brasil. Curitiba, 2007. Disponível em: <www.landaction.org/spip/spip.php?article190 - 90k>. Acesso em: 02 de ago. de 2012.

COPAS, ME; BUSSAN AJ; DRILIAS MJ; WOLKOWSKI RP. 2009. Potato yield and quality response to subsoil tillage and compaction. Agronomy Journal. 110: 82-90.

GERVÁZIO, W.; RODRIGUES C.; BESSA, G. J. L.; SILVEIRA, G. da S.; YASMAHITA, O. M. Indicadores da qualidade do solo de um agroecossistema ecológico amazônico na visão etnopedológica. Enciclopédia Biosfera, Centro Científico Conhecer - Goiânia, v.10, n.19; p2312-2326. 2014.

GLIESSMAN, S. "A agricultura pode ser sustentável". EMATER/RS. Rio Grande do Sul, 2003. Entrevista concedida a Jornalista Ângela Filippi. Disponível em: <http://www.emater.tche.br/docs/agroeco/revista/n3/03entrevista. htm. Acesso: 21 de fev.de 2014.

GUZMÁN, E, S. Agroecología y desarrollo rural sustentable: una prista. Disponivel em: https:/geografiaposgrado.files.wordpress.com/2009/04/ag roecologiaydesarrollorura1.pdf. Acesso em: 21 de fev.de 2015.

KOCHHANN, R.A.; DENARDIN, J.E.; SER TON, A.L. Compactação e descompactação de solos. Passo Fundo: Embrapa Trigo, 2000.

MACHADO, C. T. de T. Avaliação participativa do manejo de agroecossistemas e capacitação em agroecologia utilizando indicadores de sustentabilidade de 
determinação rápida e fácil. Planaltina, DF: Embrapa Cerrados, 2006.

NICHOLLS, C. I. et al. A rapid, farmer-friendly agroecological method to estimate soil quality and crop health in vineyard systems. Biodynamics, n. 250, p. 3340, 2004.

NICHOLLS, C. I.; ALTIERI, M. A.; SÁNCHEZ, E. J. Manual práctico de control biológico para una agricultura sustentable. Barcelona: Associoción Vida sana; Valencia: Sociedad Española de Agricultura Ecológica, 1999. 86 p.

OLIVEIRA, C. M. de. Desenvolvimento sustentável: uma discussão ambiental e social. In: III Jornada Internacional de Políticas Públicas São Luís - MA, 2007.

RIGO, M. M.; SILVA, V. M. da. Avaliação da qualidade do solo de um agroecossistema de café por meio de indicadores de fácil determinação. In: XV Encontro Latino Americano de Iniciação Científica e XI Encontro Latino Americano de Pós-Graduação - Universidade do Vale do Paraíba. Vale do Paraíba, 2014.

THORNTON M; STARK J; HOPKINS BG; THORNTON RE. 2008. Selecting and preparing the planting site. In: JOHNSON DA (ed). Potato health management. Saint Paul: The American Phytopathological Society. p.23-30 